\title{
NEW NORM INEQUALITIES OF ČEBYŠEV TYPE FOR POWER SERIES IN BANACH ALGEBRAS
}

\author{
S. S. DRAGOMIR ${ }^{1,2}$, M. V. BOLDEA ${ }^{3}$, AND M. MEG $\mathbb{N}^{4}$ \\ Abstract. Let $f(\lambda)=\sum_{n=0}^{\infty} \alpha_{n} \lambda^{n}$ be a function defined py power $\alpha$ ies ith \\ complex coefficients and convergent on the open disk $D, R) \subset \mathbb{C}, R>$ and \\ $x, y \in \mathcal{B}$, a Banach algebra, with $x y=y x$. In this p ablish ome new \\ upper bounds for the norm of the Čebyšev type diffe nce

$$
f(\lambda) f(\lambda x y)-f(\lambda x)(\lambda y)
$$

providing that the complex number $\lambda$ and thectors $x, \in \mathcal{B}$ are such that the series in the above expression are converge $\%$ These results oomplement the earlier resuls obtained by the authors. Applicat ns for son fundamental functions such as the exponential function and the resolv $t$ functio are provided as well.

Let $\mathcal{B}$ be an algebra. n alg
$(\mathcal{B},\|\cdot\|)$ is a normed s ace, on further

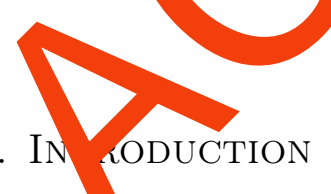

$$
\forall\|a b\| \leq\|a\|\|b\|,
$$

for any $a, b \in \gamma$. The nom algebra $(\mathcal{B},\|\cdot\|)$ is a Banach algebra if $\|\cdot\|$ is a complete norm. We oume that the banach algebra is unital, this means that $\mathcal{B}$ has an identity 1 and that $1 \| 1$.

Let a sebra. An element $a \in \mathcal{B}$ is invertible if there exists an element $b \in \mathcal{B}$ wit $-a b=a=1$. The element $b$ is unique; it is called the inverse of $a$ and writ 1 or 1 The set of invertible elements of $\mathcal{B}$ is denoted by $\operatorname{Inv} \mathcal{B}$. If $a, b \in \operatorname{Inv} \mathcal{B}$ then $a, \in \operatorname{Inv} \mathcal{B}$ and $(a b)^{-1}=b^{-1} a^{-1}$.

For a u.ital Banach algebra we also have

(i) If $a \in \mathcal{B}$ and $\lim _{n \rightarrow \infty}\left\|a^{n}\right\|^{1 / n}<1$, then $1-a \in \operatorname{Inv} \mathcal{B}$;

(ii) $\{a \in \mathcal{B}:\|1-b\|<1\} \subset \operatorname{Inv} \mathcal{B}$;

(iii) $\operatorname{Inv} \mathcal{B}$ is an open subset of $\mathcal{B}$;

\footnotetext{
Key words and phrases. Banach algebras, power series, exponential function, resolvent function, norm inequalities.

2010 Mathematics Subject Classification. Primary: 47A63. Secondary: 47A99.

Received: February 25, 2015

Accepted: April 4, 2015.
} 
(iv) The map $\operatorname{Inv} \mathcal{B} \ni a \longmapsto a^{-1} \in \operatorname{Inv} \mathcal{B}$ is continuous.

For simplicity, we denote $\lambda 1$, where $\lambda \in \mathbb{C}$ and 1 is the identity of $\mathcal{B}$, by $\lambda$. The resolvent set of $a \in \mathcal{B}$ is defined by

$$
\rho(a):=\{\lambda \in \mathbb{C}: \lambda-a \in \operatorname{Inv} \mathcal{B}\} ;
$$

the spectrum of $a$ is $\sigma(a)$, the complement of $\rho(a)$ in $\mathbb{C}$, and the resolvent function of $a$ is $R_{a}: \rho(a) \rightarrow \operatorname{Inv} \mathcal{B}, R_{a}(\lambda):=(\lambda-a)^{-1}$. For each $\lambda, \gamma \in \rho(a)$ we have the identity

$$
R_{a}(\gamma)-R_{a}(\lambda)=(\lambda-\gamma) R_{a}(\lambda) R_{a}(\gamma)
$$

We also have that $\sigma(a) \subset\{\lambda \in \mathbb{C}:|\lambda| \leq\|a\|\}$. The spectral rad $\imath_{\mathrm{a}}$ of $a$ is efined as $\nu(a)=\sup \{|\lambda|: \lambda \in \sigma(a)\}$. If $a, b$ are commuting element in $\mathcal{B}$, i.e. $a b=b a$, then

$$
\nu(a b) \leq \nu(a) \nu(b) \text { and } \nu(a+b) \leq \nu(a,+\nu, b) \text {. }
$$

Let $f$ be an analytic functions on the open disk $(0, R)$ gi $n g$ the power series $f(\lambda):=\sum_{j=0}^{\infty} \alpha_{j} \lambda^{j}(|\lambda|<R)$. If $\nu(a)<R$, then une se. ss $\sum_{j=0}^{\infty} \alpha_{j} a^{j}$ converges in the Banach algebra $\mathcal{B}$ because $\sum_{j=0}^{\infty}\left|\alpha_{j}\right|\left\|a^{j}\right\|<$ ad we ca define $f(a)$ to be its sum. Clearly $f(a)$ is well defined and there are r any examples of important functions on a Banach algebra $\mathcal{B}$ that can be constructed $n$ this we . For instance, the exponential map on $\mathcal{B}$ denoted exp and defined

$$
\exp a \cdot=\sum_{j=0}^{1} \text { tor each } a \in \mathcal{B} \text {. }
$$

If $\mathcal{B}$ is not commutative, then man the familiar properties of the exponential function from the sce ar case not hold. The following key formula is valid, however with the addition $n$ othesis commutativity for $a$ and $b$ from $\mathcal{B}$

In a gener $\operatorname{Ban} \boldsymbol{A}$ algebra $\mathcal{B}$ it is difficult to determine the elements in the range of the exponent map $\uparrow(\mathcal{B})$, i.e., the element which have a "logarithm". However, it is e? $y$ to ee tho $a$ is an element in $B$ such that $\|1-a\|<1$, then $a$ is in $\exp (\mathcal{B})$. Th t foll wom the fact that if we set

$$
b=-\sum_{n=1}^{\infty} \frac{1}{n}(1-a)^{n},
$$

then the series converges absolutely and, as in the scalar case, substituting this series into the series expansion for $\exp (b)$ yields $\exp (b)=a$.

It is known that if $x$ and $y$ are commuting, i.e., $x y=y x$, then the exponential function satisfies the property $\exp (x) \exp (y)=\exp (y) \exp (x)=\exp (x+y)$. Also, if $x$ is invertible and $a, b \in \mathbb{R}$ with $a<b$ then

$$
\int_{a}^{b} \exp (t x) d t=x^{-1}[\exp (b x)-\exp (a x)] .
$$


Moreover, if $x$ and $y$ are commuting and $y-x$ is invertible, then

$$
\begin{aligned}
\int_{0}^{1} \exp ((1-s) x+s y) d s & =\int_{0}^{1} \exp (s(y-x)) \exp (x) d s \\
& =\left(\int_{0}^{1} \exp (s(y-x)) d s\right) \exp (x) \\
& =(y-x)^{-1}[\exp (y-x)-I] \exp (x) \\
& =(y-x)^{-1}[\exp (y)-\exp (x)
\end{aligned}
$$

Inequalities for functions of operators in Hilbert spaces may be und in t e papers [3], [2] and in the recent monographs [4], [5], [7] and the ref ences ereip

In order to state some earlier results [6] that motivat our crent ork we need some preparation as follows.

Let $\alpha_{n}$ be nonzero complex numbers and let

$$
R:=\frac{1}{\operatorname{lims} \rho\left|\alpha_{n}\right|^{\frac{1}{n}}} .
$$

Clearly $0 \leq R \leq \infty$, but we consider only he case $0<R \leq \infty$.

Denote by

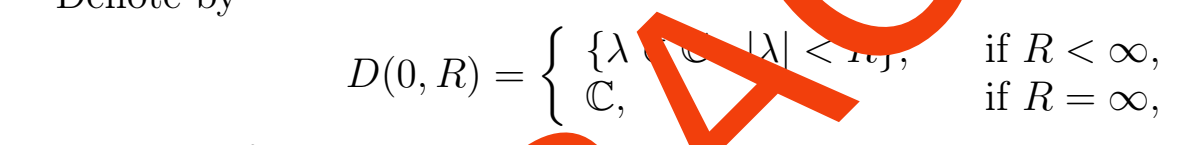

consider the functions

and

$$
\lambda f\left(0, \text { ho } \rightarrow \mathbb{C}, \quad f(\lambda):=\sum_{n=0}^{\infty} \alpha_{n} \lambda^{n}\right.
$$

$$
\nu \mapsto f_{A}(\lambda): D(0, R) \rightarrow \mathbb{C}, \quad f_{A}(\lambda):=\sum_{n=0}^{\infty}\left|\alpha_{n}\right| \lambda^{n} .
$$

$$
\begin{aligned}
& \text { Let a a a nebra and } 1 \text { its unity. Denote by } \\
& B(0, R)= \begin{cases}\{x \in \mathcal{B}:\|x\|<R\}, & \text { if } R<\infty \\
\mathcal{B}, & \text { if } R=\infty\end{cases}
\end{aligned}
$$

We asso ate to $f$ the map

$$
x \mapsto \widetilde{f}(x): B(0, R) \rightarrow \mathcal{B}, \quad \widetilde{f}(x):=\sum_{n=0}^{\infty} \alpha_{n} x^{n} .
$$

Obviously, $\tilde{f}$ is correctly defined because the series $\sum_{n=0}^{\infty} \alpha_{n} x^{n}$ is absolutely convergent, since $\sum_{n=0}^{\infty}\left\|\alpha_{n} x^{n}\right\| \leq \sum_{n=0}^{\infty}\left|\alpha_{n}\right|\|x\|^{n}$.

In addition, we assume that $s_{2}:=\sum_{n=0}^{\infty} n^{2}\left|\alpha_{n}\right|<\infty$. Let $s_{0}:=\sum_{n=0}^{\infty}\left|\alpha_{n}\right|<\infty$ and $s_{1}:=\sum_{n=0}^{\infty} n\left|\alpha_{n}\right|<\infty$.

With the above assumptions we have the following [6]. 
Theorem 1.1. Let $\lambda \in \mathbb{C}$ such that $\max \left\{|\lambda|,|\lambda|^{2}\right\}<R<\infty$ and let $x, y \in \mathcal{B}$ with $\|x\|,\|y\| \leq 1$ and $x y=y x$. Then

(i) We have

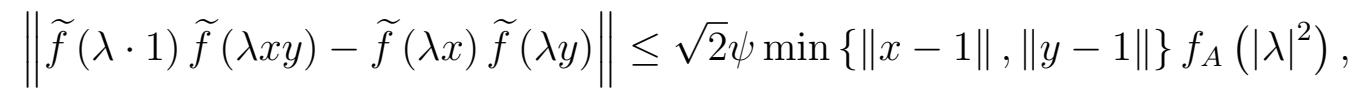
where

$$
\psi^{2}:=s_{0} s_{2}-s_{1}^{2} .
$$

(ii) We also have

$$
\begin{aligned}
& \|\tilde{f}(\lambda \cdot 1) \tilde{f}(\lambda x y)-\tilde{f}(\lambda x) \tilde{f}(\lambda y)\| \leq \sqrt{2} \min \{\|x-1 /\| y>(\mid \|\} f \mid) \\
& \quad \times\left\{f_{A}(|\lambda|)\left[|\lambda| f_{A}^{\prime}(|\lambda|)+|\lambda|^{2} f_{A}^{\prime \prime}(|\lambda|)\right]-\left[|\lambda| \lambda\left(\left|\lambda^{\prime}\right|\right\}^{1 / 2}\right.\right.
\end{aligned}
$$

For other similar results, see [6].

In this paper we establish some new upper bourds to the norm of the Čebyšev type difference

$$
\widetilde{f}(\lambda \cdot 1) \widetilde{f}(\lambda x y)-\widetilde{f}(\lambda x) \widetilde{\widetilde{N}}(\lambda y)
$$

provide that the complex number $\lambda$ and the vectors , $y \in \mathcal{B}$ are such that the series in (1.4) are convergent. Applicatic or som andamental functions such as the exponential function and the resolve, fur are provided as well.

\section{Th Results}

We start with the fo "ow c csult wat is of interest in itself.

Lemma 2.1. Let $\mathcal{N}=\sum_{n} \alpha_{n} \lambda^{n}$ be a function defined by power series with complex coefficients and onvergent on the open disk $D(0, R) \subset \mathbb{C}, R>0$ and $x, y \in \mathcal{B}$ with $x y=y \quad$ If $\|y\|<\lambda \in \mathbb{C}$ and $x \in \mathcal{B}$ with $|\lambda|\|x\|<R$, then we have the inequality

$$
\left\|f\left(v_{y}\right) y^{k}-\tilde{f}(\lambda x y)\right\| \leq \frac{\|y-1\|}{1-\|y\|}\left[f_{A}(|\lambda|\|x\|)-\left|\alpha_{k}\right||\lambda|^{k}\|x\|^{k}\right]
$$

for $n y \in \mathbb{N}, \geq 0$.

Proof. have for $m \geq 2$ and $1 \leq k \leq m-1$ that

$$
\begin{aligned}
\left(\sum_{j=0}^{m} \alpha_{j} \lambda^{j} x^{j}\right) y^{k}-\sum_{j=0}^{m} \alpha_{j} \lambda^{j}(x y)^{j} & =\left(\sum_{j=0}^{m} \alpha_{j} \lambda^{j} x^{j}\right) y^{k}-\sum_{j=0}^{m} \alpha_{j} \lambda^{j} x^{j} y^{j} \\
& =\sum_{j=0}^{m} \alpha_{j} \lambda^{j} x^{j}\left(y^{k}-y^{j}\right) \\
& =\sum_{j=0, j \neq k}^{m} \alpha_{j} \lambda^{j} x^{j}\left(y^{k}-y^{j}\right)=A .
\end{aligned}
$$


Since $y^{k}-y^{j}=\sum_{l=j}^{k-1}\left(y^{l-1}-y^{l}\right)=\sum_{l=j}^{k-1} y^{l}(y-1)$, then by taking the norm in (2.2) we get

$$
\begin{aligned}
\|A\| & \leq \sum_{j=0, j \neq k}^{m}\left|\alpha_{j}\right||\lambda|^{j}\|x\|^{j}\left\|\sum_{l=j}^{k-1} y^{l}(y-1)\right\| \\
& \leq \sum_{j=0, j \neq k}^{m}\left|\alpha_{j}\right||\lambda|^{j}\|x\|^{j} \sum_{l=j}^{k-1}\|y\|^{l}\|y-1\| \\
& =\|y-1\| \sum_{j=0, j \neq k}^{m}\left|\alpha_{j}\right||\lambda|^{j}\|x\|^{j} \sum_{l=j}^{k-1}\|y\|^{l}=: B .
\end{aligned}
$$

By noticing that

we have

$$
\begin{aligned}
B & \leq\|y-1\| \sum_{l=0}^{m-1}\|y\|^{l} \sum_{j=k}^{m}\left|\alpha_{j}\right| \lambda \lambda_{1}, x^{\prime} \|^{j} \\
& \left.=\|y-1\| \sum_{0}^{m}\left\|\left.y|| \lambda\right|^{j}\right\| x\left\|^{j}-\left|\alpha_{k}\right||\lambda|^{k}\right\| x \|^{k}\right) .
\end{aligned}
$$

Utilising the ine a ries $(2.2,(2.4)$ we conclude that

$$
\begin{aligned}
& \left\|\left(\sum_{j=0}^{m} \alpha_{j} \lambda^{j} x^{\prime}\right) y^{k}-\sum_{j=0}^{m} \alpha_{j} \lambda^{j}(x y)^{j}\right\|
\end{aligned}
$$

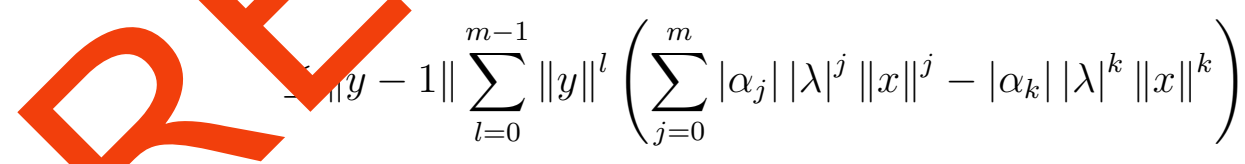

for any $\geq 2$ and $1 \leq k \leq m-1$.

Since the series $\sum_{j=0}^{m} \alpha_{j} \lambda^{j} x^{j}$ and $\sum_{j=0}^{m} \alpha_{j}(\lambda x y)^{j}$ are convergent in $\mathcal{B}$ and, because $\|y\|<1$, then $\sum_{l=0}^{\infty}\|y\|^{l}=\frac{1}{1-\|y\|}$, then by letting $m \rightarrow \infty$ in (2.5), we get the desired result $(2.1)$.

If $k=0$, then $\sum_{j=0}^{m} \alpha_{j} \lambda^{j} x^{j}-\sum_{j=0}^{m} \alpha_{j} \lambda^{j}(x y)^{j}=\sum_{j=1}^{m} \alpha_{j} \lambda^{j} x^{j}\left(1-y^{j}\right)=: C$. Since $1-y^{j}=(1-y)\left(1+y+\ldots+y^{j-1}\right), j \geq 1$, then

$$
\left\|1-y^{j}\right\| \leq\|y-1\| \sum_{l=0}^{j-1}\|y\|^{l} \leq\|y-1\| \sum_{l=0}^{m-1}\|y\|^{l}
$$


and then

$$
\begin{aligned}
\|C\| & \leq\|y-1\| \sum_{l=0}^{m-1}\|y\|^{l} \sum_{j=1}^{m}\left|\alpha_{j}\right||\lambda|^{j}\|x\|^{j} \\
& =\|y-1\| \sum_{l=0}^{m-1}\|y\|^{l}\left(\sum_{j=0}^{m}\left|\alpha_{j}\right||\lambda|^{j}\|x\|^{j}-\left|\alpha_{0}\right|\right) .
\end{aligned}
$$

Letting $m \rightarrow \infty$ in (2.6), we also obtain the inequality (2.1) for $k-$ This proves the lemma.

Corollary 2.1. Let $f(\lambda)=\sum_{n=0}^{\infty} \alpha_{n} \lambda^{n}$ be a function defind by ower $s$ ries with complex coefficients and convergent on the open disk $D(0, \mathcal{C} \subset \mathbb{C}, h \vee$ and $x \in \mathcal{B}$. If $\|x\|<1, \lambda \in \mathbb{C}$ with $|\lambda|\|x\|<R$, then we have the in yalit

$$
\left\|\tilde{f}(\lambda x) x^{k}-\tilde{f}\left(\lambda x^{2}\right)\right\| \leq \frac{\|x-1\|}{1-\|x\|}\left[f_{A}(\mid \gamma, x \|)-\mid \alpha_{k}, y /\|x\|^{k}\right],
$$

for any $k \in \mathbb{N}, k \geq 0$.

We can state the following result.

Theorem 2.1. Let $f(\lambda)=\sum_{n=0}^{\infty} \alpha_{n} \lambda^{n}$ be functi $n$ defined by power series with complex coefficients and convergent opert $D(0, R) \subset \mathbb{C}, R>0$ and $x, y \in \mathcal{B}$ with $x y=y x$. If $\lambda, \mu \in \mathbb{C}$ are such th $t \mid \forall \|<R$ and $\|y\| \leq 1$ then

$$
\begin{aligned}
& \| \tilde{f}(\lambda x) \tilde{f}(g)-\tilde{f}(\mu \cdot \widetilde{f}(\lambda x y) \| \\
& \left.\quad \leq \frac{\|-\| y \|}{A} f_{A}(|\lambda|\|x\|) f_{A}(|\mu|)-f_{A^{2}}(|\lambda||\mu|\|x\|)\right],
\end{aligned}
$$

where $f_{A^{2}}(\lambda):=\sum_{n=0}^{\infty} \lambda V^{2} \lambda^{n}$.

Proof. Utili ag Lenma 2.1 Wwe have

$$
\begin{aligned}
& =\left\|\sum_{k=0}^{p} \alpha_{k} \mu^{k}\left(\tilde{f}(\lambda x) y^{k}-\tilde{f}(\lambda x y)\right)\right\| \\
& \leq \sum_{k=0}^{p}\left|\alpha_{k}\right||\mu|^{k}\left\|\tilde{f}(\lambda x) y^{k}-\tilde{f}\left(\sum_{k=0}^{p} \alpha_{k} \mu^{k}\right) \tilde{f}(\lambda x y)\right\| \\
& \leq \sum_{k=0}^{p} \frac{\|y-1\|}{1-\|y\|}\left[f_{A}(|\lambda|\|x\|)-\left|\alpha_{k}\right||\lambda|^{k}\|x\|^{k}\right]\left|\alpha_{k}\right||\mu|^{k} \\
& =\frac{\|y-1\|}{1-\|y\|}\left[f_{A}(|\lambda|\|x\|) \sum_{k=0}^{p}\left|\alpha_{k}\right||\mu|^{k}-\sum_{k=0}^{p}\left|\alpha_{k}\right||\lambda|^{k}|\mu|^{k}\|x\|^{k}\right]
\end{aligned}
$$


for any $p \geq 0$.

Since all the series that are involved in the inequality from above are convergent, then by letting $p \rightarrow \infty$ we get the desired result (2.7).

Corollary 2.2. Let $f(\lambda)=\sum_{n=0}^{\infty} \alpha_{n} \lambda^{n}$ be a function defined by power series with complex coefficients and convergent on the open disk $D(0, R) \subset \mathbb{C}, R>0$ and $x \in \mathcal{B}$. If $\lambda, \mu \in \mathbb{C}$ are such that $|\mu|,|\lambda|\|x\|<R$ and $\|x\|<1$ then

$$
\begin{aligned}
& \left\|\tilde{f}(\lambda x) \tilde{f}(\mu x)-\tilde{f}(\mu \cdot 1) \tilde{f}\left(\lambda x^{2}\right)\right\| \\
& \quad \leq \frac{\|x-1\|}{1-\|x\|}\left[f_{A}(|\lambda|\|x\|) f_{A}(|\mu|)-f_{A^{2}}\left(|\lambda||\mu| \| x_{\mid}\right.\right.
\end{aligned}
$$

Remark 2.1. If $\mu=\lambda$, then we get the inequality for the reby $\sim$ functronal $\|\tilde{f}(\lambda x) \tilde{f}(\lambda y)-\tilde{f}(\lambda \cdot 1) \tilde{f}(\lambda x y)\| \leq \frac{\|y-1\|}{1-\|y\|}\left[f_{A}(\|x\|) f_{A} \vee V-f_{A^{2}}\left(|\lambda|^{2}\|x\|\right)\right]$, provided that $x, y \in \mathcal{B}$ with $x y=y x, \lambda \in$ are such that $|\lambda|,|\lambda|\|x\|<R$ and $\|y\|<1$. From $(2.8)$ we have

$$
\begin{aligned}
& \|[\tilde{f}(\lambda x)]^{2}-\tilde{f}(\lambda \cdot 1) \tilde{f}(\lambda x \\
& \quad \leq \frac{\|x-1\|}{1-\|x\|}\left[f_{4}\left(|\lambda|, \quad \| f_{A}(|\mu|)-f_{A^{2}}(|\lambda||\mu|\|x\|)\right] .\right.
\end{aligned}
$$

We can state now the cond

Theorem 2.2. Let $(\lambda)=\Delta_{=0}^{\infty} \alpha_{n} \lambda^{n}$ be a power series that is convergent on the open disk $D(0, R$ wa $R>0$. If $x, y \in \mathcal{B}$ with $x y=y x$ and $\|y\|,\|y\| \leq 1$, then we have the inequ ities

$$
\begin{aligned}
& \tilde{f}(\lambda \wedge \tilde{f}(\lambda x y)-\tilde{f}(\lambda x) \tilde{f}(\lambda y) \| \\
& f_{A}(\lambda):=\sum_{n=0}^{\infty}\left|\alpha_{n}\right| \lambda^{n}, g_{A}(\lambda):=\sum_{n=0}^{\infty} n^{4}\left|\alpha_{n}\right| \lambda^{n}, h_{A}(\lambda):=\sum_{n=0}^{\infty} n^{2}\left|\alpha_{n}\right| \lambda^{n}
\end{aligned}
$$

and $\lambda \in D(0, R)$.

Moreover, if the series $s_{0}:=\sum_{n=0}^{\infty}\left|\alpha_{n}\right|, s_{2}:=\sum_{n=0}^{\infty} n^{2}\left|\alpha_{n}\right|$ and $s_{4}:=\sum_{n=0}^{\infty} n^{4}\left|\alpha_{n}\right|$ are convergent, then we have the inequalities

$$
\|\tilde{f}(\lambda x) \tilde{f}(\lambda y)-\tilde{f}(\lambda \cdot 1) \tilde{f}(\lambda x y)\| \leq \frac{\sqrt{2}}{2}\|x-1\|\|y-1\| f_{A}\left(|\lambda|^{2}\right)\left[s_{0} s_{4}-s_{2}^{2}\right]^{\frac{1}{2}},
$$

for any $\lambda \in \mathbb{C}$ with $|\lambda|,|\lambda|^{2}<R$. 
Proof. We observe that

$$
\begin{aligned}
B_{m}:= & \sum_{n, j=0}^{m} \alpha_{n} \alpha_{j} \lambda^{n} \lambda^{j}\left(x^{n}-x^{j}\right)\left(y^{n}-1\right) \\
= & \sum_{n, j=0}^{m} \alpha_{n} \alpha_{j} \lambda^{n} \lambda^{j}\left(x^{n} y^{n}-x^{j} y^{n}-x^{n}+x^{j}\right) \\
= & \sum_{j=0}^{m} \alpha_{j} \lambda^{j} \sum_{n=0}^{m} \alpha_{n} \lambda^{n}(x y)^{n}-\sum_{j=0}^{m} \alpha_{j} \lambda^{j} x^{j} \sum_{n=0}^{m} \alpha_{n} y^{n} y^{n} \\
& -\sum_{j=0}^{m} \alpha_{j} \lambda^{j} \sum_{n=0}^{m} \alpha_{n} \lambda^{n} x^{n}+\sum_{j=0}^{m} \alpha_{j} \lambda^{j} x^{j} \sum_{=0}^{m} \alpha_{n} \lambda^{n} \\
= & \sum_{j=0}^{m} \alpha_{j} \lambda^{j} \sum_{n=0}^{m} \alpha_{n} \lambda^{n}(x y)^{n}-\sum_{j=0}^{m} \lambda^{j} x^{j} \sum_{n=0} y_{n}^{n} .
\end{aligned}
$$

Taking the norm and using the generalize ongle ine ality we have:

$$
\left.\left\|B_{m}\right\| \leq \sum_{n, j=0}^{m}\left|\alpha_{n}\right|\left|\alpha_{j}\right||\lambda|^{n}|\lambda| \| x^{n}-x^{3}\right)\left\|y^{n}-1\right\|:=C_{m} .
$$

Since $y^{n}-1=(y-1)\left(y^{n-1}+\ldots+1\right.$ we wor $\|y\| \leq 1$ that

$$
\left\|y^{n}-1\right\|<\text { त9 } 1\|\|^{-1}+\ldots+1\|\leq n\| y-1 \| \text {. }
$$

If $n>j$, then for $\|x\| \leq$

$$
\left\|x^{n}-x^{j}\right\|=\sqrt{ } x^{j}(x-1)\|\leq\| x\left\|^{j}\right\| x^{n-j}-1\|\leq(n-j)\| x-1 \| .
$$

Similarly, if $j>$ we ve $\left\|x^{n}-x^{j}\right\| \leq(j-n)\|x-1\|$. Therefore for any $n, j \in \mathbb{N}$ we have

\section{Utilising this fo ws we}

$$
\left\|x^{n}-x^{j}\right\| \leq|n-j|\|x-1\|,\|x\| \leq 1 .
$$

$$
\begin{aligned}
C_{m} & \leq \sum_{n, j=0}^{m}\left|\alpha_{n}\right|\left|\alpha_{j}\right||\lambda|^{n}|\lambda|^{j} n|n-j|\|x-1\|\|y-1\| \\
& =\|x-1\|\|y-1\| \sum_{n, j=0}^{m}\left|\alpha_{n}\right|\left|\alpha_{j}\right||\lambda|^{n}|\lambda|^{j} n|n-j| .
\end{aligned}
$$

Further, observe that

$$
\begin{aligned}
\sum_{n, j=0}^{m}\left|\alpha_{n}\right|\left|\alpha_{j}\right||\lambda|^{n}|\lambda|^{j} n|n-j| & =\frac{1}{2} \sum_{n, j=0}^{m}\left|\alpha_{n}\right|\left|\alpha_{j}\right||\lambda|^{n}|\lambda|^{j}|n-j|(n+j) \\
& =\frac{1}{2} \sum_{n, j=0}^{m}\left|\alpha_{n}\right|\left|\alpha_{j}\right||\lambda|^{n}|\lambda|^{j}\left|n^{2}-j^{2}\right|,
\end{aligned}
$$


therefore

$$
C_{m} \leq \frac{1}{2}\|x-1\|\|y-1\| \sum_{n, j=0}^{m}\left|\alpha_{n}\right|\left|\alpha_{j}\right||\lambda|^{n}|\lambda|^{j}\left|n^{2}-j^{2}\right|:=D_{m} .
$$

Using Cauchy-Bunyakovsky-Schwarz inequality we have

$$
\begin{aligned}
& \sum_{n, j=0}^{m}\left|\alpha_{n}\right|\left|\alpha_{j}\right||\lambda|^{\frac{n}{2}}|\lambda|^{\frac{j}{2}}|\lambda|^{\frac{n}{2}}|\lambda|^{\frac{j}{2}}\left|n^{2}-j^{2}\right| \\
& \quad \leq\left(\sum_{n, j=0}^{m}\left|\alpha_{n}\right|\left|\alpha_{j}\right||\lambda|^{n}|\lambda|^{j}\right)^{\frac{1}{2}}\left(\sum_{n, j=0}^{m}\left|\alpha_{n}\right|\left|\alpha_{j}\right||\lambda|^{n}|\lambda|^{j}\left(n-j^{2}\right)^{2}\right) \\
& \quad=\left(\sum_{n=0}^{m}\left|\alpha_{n}\right||\lambda|^{n}\right)\left(E_{m}\right)^{\frac{1}{2}}
\end{aligned}
$$

where

$$
\begin{aligned}
E_{m} & :=\sum_{n, j=0}^{m}\left|\alpha_{n}\right|\left|\alpha_{j}\right||\lambda|^{n}|\lambda|^{j}\left(n^{2}-j^{2}\right)^{2} \\
& =\sum_{n, j=0}^{m}\left|\alpha_{n}\right|\left|\alpha_{j}\right||\lambda|^{n}\left(n^{4}-2^{4}\right)^{2} \\
& =2\left[\left.\sum_{n \rightarrow 0}^{m}|n||\lambda|^{n} \sum_{n=0}^{m}\left|\alpha_{i}\right| \lambda\right|^{n} n^{4}-\left(\sum_{n=0}^{m}\left|\alpha_{n}\right||\lambda|^{n} n^{2}\right)^{2}\right] .
\end{aligned}
$$

Making use of $(2-(2.13)$ het for $\|x\|,\|y\| \leq 1$ that

$$
\begin{aligned}
& \text { 2.14) } \\
& \times\left[\left.\sum_{i=0}^{m}\left|\alpha_{j} \lambda^{j} \sum_{n=0}^{m} \alpha_{n}(\lambda x y)^{n}-\sum_{j=0}^{m} \alpha_{j}(\lambda x)^{j} \sum_{n=0}^{m} \alpha_{n=0}^{m} \alpha^{4}\right| \alpha_{n}|| \lambda\right|^{n}-\left(\sum_{n=0}^{m} n^{2}\left|\alpha_{n}\right||\lambda|^{n}\right)^{2}\right]^{\frac{1}{2}}, \\
& \times\left[y-1 \| \sum_{n=0}^{m}\left|\alpha_{n}\right||\lambda|^{n}\right.
\end{aligned}
$$

for any $m \in \mathbb{N}$.

Since all the series involved in (2.14) are convergent, then by letting $m \rightarrow \infty$ in (2.14) we deduce the desired result

$$
\begin{aligned}
& \|\tilde{f}(\lambda \cdot 1) \tilde{f}(\lambda x y)-\tilde{f}(\lambda x) \tilde{f}(\lambda y)\| \\
& \quad \leq \frac{\sqrt{2}}{2}\|x-1\|\|y-1\| f_{A}(|\lambda|)\left[f_{A}(|\lambda|) g_{A}(|\lambda|)-h_{A}^{2}(|\lambda|)\right]^{\frac{1}{2}} .
\end{aligned}
$$


Using Cauchy-Bunyakovsky-Schwarz inequality we also have

$$
\begin{aligned}
& \sum_{n, j=0}^{m}\left|\alpha_{n}\right|\left|\alpha_{j}\right||\lambda|^{n}|\lambda|^{j}\left|n^{2}-j^{2}\right| \\
& \quad \leq\left(\sum_{n, j=0}^{m}\left|\alpha_{n}\right|\left|\alpha_{j}\right||\lambda|^{2 n}|\lambda|^{2 j}\right)^{\frac{1}{2}}\left(\sum_{n, j=0}^{m}\left|\alpha_{n}\right|\left|\alpha_{j}\right|\left|n^{2}-j^{2}\right|^{2}\right)^{\frac{1}{2}} \\
& \quad=\left(\sum_{n=0}^{m}\left|\alpha_{n}\right||\lambda|^{2 n}\right)\left\{2\left[\sum_{n=0}^{m}\left|\alpha_{n}\right| \sum_{j=0}^{m} j^{4}\left|\alpha_{j}\right|-\left(\sum_{n=}^{m} n^{2} \mid\right)^{2}\right]\right\}^{\frac{1}{2}}
\end{aligned}
$$

Making use of this inequality we then obtain in a sin ar w the sucond part of the theorem. The details are omitted.

\section{Some Examples} Then

Consider the function $f: D(0,1) \rightarrow \mathbb{C}$ defined by $f(\lambda)=(1-\lambda)^{-1}=\sum_{k=0}^{\infty} \lambda^{k}$.

and by (2.7), we have for,$y=\mathcal{B}$ ith $x y=y x,\|y\|<1$ and $\lambda, \mu \in \mathbb{C}$ with $|\mu|,|\lambda|\|x\|<1$ that

$$
\begin{aligned}
& \left\|(1-\lambda x)(1-\lambda)^{-1}-(1-\mu)^{-1}(1-\lambda x y)^{-1}\right\| \\
& \left.\zeta \frac{\|-\| y \|}{1-\|}(1-|\lambda|\|x\|)^{-1}(1-|\mu|)^{-1}-(1-|\lambda||\mu|\|x\|)^{-1}\right] .
\end{aligned}
$$

In particur if $\mid,\|x\|<1$, then

$$
\begin{aligned}
& \|\left(1-\lambda(1-\lambda y)^{-1}-(1-\lambda)^{-1}(1-\lambda x y)^{-1} \|\right. \\
& \frac{\|y-1\|}{1-\|y\|}\left[(1-|\lambda|\|x\|)^{-1}(1-|\lambda|)^{-1}-\left(1-|\lambda|^{2}\|x\|\right)^{-1}\right] .
\end{aligned}
$$

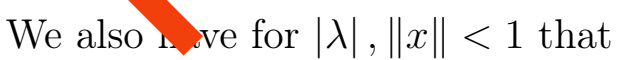

$$
\begin{aligned}
& \left\|(1-\lambda x)^{-2}-(1-\lambda)^{-1}\left(1-\lambda x^{2}\right)^{-1}\right\| \\
& \quad \leq \frac{\|x-1\|}{1-\|x\|}\left[(1-|\lambda|\|x\|)^{-1}(1-|\lambda|)^{-1}-\left(1-|\lambda|^{2}\|x\|\right)^{-1}\right] .
\end{aligned}
$$

If we consider the function $f(\lambda)=(1+\lambda)^{-1}=\sum_{k=0}^{\infty}(-1)^{k} \lambda^{k}$, then the inequalities (3.1)-(3.3) also hold with "+" instead of "-" in the left hand side expressions such as $(1-\lambda x)^{-1}$ etc. 
We consider the modified Bessel function functions of the first kind

$$
I_{\nu}(\lambda):=\left(\frac{1}{2} \lambda\right)^{\nu} \sum_{k=0}^{\infty} \frac{\left(\frac{1}{4} \lambda^{2}\right)^{k}}{k ! \Gamma(\nu+k+1)}, \lambda \in C
$$

where $\Gamma$ is the Gamma function and $\nu$ is a real number. An integral formula is

$$
I_{\nu}(\lambda)=\frac{1}{\pi} \int_{0}^{\pi} e^{\lambda \cos \theta} \cos (\nu \theta)-\frac{\sin (\nu \pi)}{\pi} \int_{0}^{\infty} e^{-\lambda \cosh t-\nu t} d t
$$

which simplifies for $\nu$ an integer $n$ to [1]

$$
I_{n}(\lambda)=\frac{1}{\pi} \int_{0}^{\pi} e^{\lambda \cos \theta} \cos (n \theta) d
$$

For $n=0$ we have $I_{0}(\lambda)=\frac{1}{\pi} \int_{0}^{\pi} e^{\lambda \cos \theta} d \theta=\sum_{k=\frac{4}{(k !)}}^{\infty}, \lambda \in C$

Now, if we consider the exponential functi $(\lambda)=\mathrm{e} \lambda(\lambda)=\sum_{k=0}^{\infty} \frac{1}{k !} \lambda^{k}$, then for $\rho>0$ we have

$$
\left.f_{A^{2}}(\rho)=\sum_{=0}^{\infty} \frac{1}{(k !)^{2}} k=I_{0}\right)(\sqrt{\rho}) .
$$

Making use of the inequality (2.7), w ve lor $x, y \in \mathcal{B}$ with $x y=y x,\|y\|<1$ and $\lambda, \mu \in \mathbb{C}$ that

$$
\begin{aligned}
& \|\exp (\lambda 2+\mu) \exp (-y+\mu \cdot 1)\| \\
& 2 \frac{1-1 \|}{1-x \|}\left[\operatorname{ex}(|\lambda|\|x\|+|\mu|)-I_{0}(2 \sqrt{|\lambda||\mu|\|x\|})\right] .
\end{aligned}
$$

In particulg, we brve

$$
\begin{aligned}
& \leq \exp (\lambda+y))-\exp (\lambda(x y+1)) \| \\
& \leq \frac{\|y-1\|}{1-\|y\|}\left[\exp (|\lambda|(\|x\|+1))-I_{0}(2|\lambda| \sqrt{\|x\|})\right] .
\end{aligned}
$$

We also ave for $\|x\|<1$

$$
\left\|\exp (2 \lambda x)-\exp \left(\lambda\left(x^{2}+1\right)\right)\right\| \leq \frac{\|x-1\|}{1-\|x\|}\left[\exp (|\lambda|(\|x\|+1))-I_{0}(2|\lambda| \sqrt{\|x\|})\right]
$$

for any $\lambda \in \mathbb{C}$. If we take $\lambda=1$, then we get

$$
\left\|\exp (2 x)-\exp \left(x^{2}+1\right)\right\| \leq \frac{\|x-1\|}{1-\|x\|}\left[\exp (\|x\|+1)-I_{0}(2 \sqrt{\|x\|})\right]
$$

for $\|x\|<1$. 


\section{REFERENCES}

[1] M. Abramowitz and I. A. Stegun (Eds.). Modified Bessel Functions I and K. §9.6 in Handbook of Mathematical Functions with Formulas, Graphs, and Mathematical Tables, 9th printing. New York: Dover, pp. 374-377, 1972.

[2] S. S. Dragomir, Inequalities for the Čebyšev functional of two functions of selfadjoint operators in Hilbert spaces, Aust. J. Math. Anal. \& Appl. 6 (2009), Issue 1, Article 7, 1-58.

[3] S. S. Dragomir, Some inequalities for power series of selfadjoint operators in Hilbert spaces via reverses of the Schwarz inequality, Integral Transforms Spec. Funct. 20 (2009), no. 9-10, 757-767.

[4] S. S. Dragomir, Operator Inequalities of the Jensen, Čebyšev and Grüss Tyn inger Briefs in Mathematics. Springer, New York, 2012. xii+121 pp. ISBN: 978-1-4614-1 $20-6$.

[5] S. S. Dragomir, Operator Inequalities of Ostrowski and Trapezoidal $\mathrm{\Lambda}$ e. Spring Briefs in Mathematics. Springer, New York, 2012. x+112 pp. ISBN: 978-1-4f 4-177

[6] S. S. Dragomir, M. V. Boldea, C. Buşe and M. Megan, Norm equalities o, Ǧe ssev type for power series in Banach algebras, J. Inequal. Appl. 2014, 294 (14), 1 pp.

[7] T. Furuta, J. Mićić Hot, J. Pečarić and Y. Seo, Mond-Peč ić $M^{\prime} d$ in O rator Inequalities. Inequalities for Bounded Selfadjoint Operators on a Hilb Space, b men Zagreb, 2005.

${ }^{1}$ Mathematics, School of Engineering \& Science

ViCTORIA University, PO Box 14428

Melbourne City, MC 8001,

Australia.

E-mail address: sever.dragomir@vu.edu.au

$U R L$ : http://rgmia.org/dragomir

${ }^{2}$ School of Computational \& Appl D A Matics,

UNIVERSiTy OF THE WitWAT 2 ND,

Private Bag 3, Johanné URG 2 50,

South AFrica

${ }^{3}$ Mathematics and Tatistio

Banat Universy a Agricul ral Sciences and Veterinary Medicine Timişoara, 119 Calea Apydului, 0645 Timişoara,

ROMÂNIA

${ }^{4}$ Departi vi Mathematics,

WESTITNIVL ITY O' I'IMIŞOARA,

B UL PÂR V , 1900-TimişOARA, 